\title{
Comparative Study on Multi-focus Image Fusion Techniques in Dynamic Scene
}

\author{
Rajvi Patel \\ Research Scholar, CSE \\ Department \\ Parul Institute of \\ Technology,Limbda. \\ Vadodara, Gujarat.
}

\author{
Manali Rajput \\ Asst. Prof, CSE Department \\ Parul Institute of \\ Technology,Limbda. \\ Vadodara, Gujarat.
}

\author{
Pramit Parekh \\ Research scholar, IT \\ Department \\ Chandubhai S. Patel Institute of \\ Technology, Changa. \\ Anand, Gujarat.
}

\begin{abstract}
In this paper, study of various image fusion techniques especially for multi-focus images has been introduced. With the increased development of technology, it is necessary to retrieve information from multi source images in order to produce a high quality fused image with spatial and spectral information. Image Fusion is the process that allows the combination of the significant information from a bunch of images into a single image, where the resultant fused image will be more qualitable informative than any other input images. Thus this technique is useful in improving the quality of data in images. Important applications of Image Fusion contain medical imaging, remote sensing, microscopic imaging, computer vision and robotics applications. Even if the fused image can have balancing spatial and spectral resolution characteristics, the existing image fusion techniques can distort the sprectral information of the multisprectal data while merging.
\end{abstract}

\section{Keywords}

Multi-focus image fusion; Spatial domain fusion; Transform domain fusion;

\section{INTRODUCTION}

Generally, several images of the same scene can be obtained to enhance the robustness of image processing system. However, Series of images for viewing and analyzing are not convenient and efficient. For that image fusion techniques are effective solution to resolve this problem. Image Fusion Techniques combined the complementary, necessary and required information from multiple images into a fused image, which is very useful for human or machine perception.

Today, many image fusion algorithms have been developed and used in many applications to merge multi-focus information into single composite image. We can consider these image fusion algorithms as classified in two broad categories: Transform domain fusion and spatial domain fusion [2]. The most commonly used transform domain fusion techniques are DCT (Discrete Cosine Transform), DWT (Discrete Wavelet Transform), Pyramid Method, RDWT (Redundant DWT), SWT (Stationary Wavelet Transform), DT-CWT (Dual tree complex w) and so on. Recently, some novel transform domain analysis methods, such as curvelet transform, contourlet transform, log-Gabor wavelet transform, support value transform, retina-inspired model and sparse representation are also applied to image fusion [1]. Usually, the transform domain methods perform three common steps. First, Input Images are converted into transform domain to get corresponding transform coefficients. Then transform coefficients are merged together according to defined fusion rule. Finally, the fused resultant image is obtained by performing inverse transform on merged coefficient. Unlike the transform domain methods, spatial domain fusion methods perform fusion process in spatial domain directly. Basically, spatial domain fusion method divided into two categories: Pixel level fusion methods and Region or feature level fusion methods. The fundamental idea behind these two methods is to clearer the final resultant image.

In general, Traditional Multi-focus image fusion Techniques provide satisfactory result on static scenes. But researcher found problem with the multi-focus image fusion in dynamic scene with camera movements and object motion. For that reasons the contents in the same position of multiple images may be different from each other. With this type of multifocus images the transform domain fusion techniques usually suffer from artifacts in the fused image because of inconsistent of image contents. In Spatial domain fusion the focus information estimated by spatial variance, image gradient or spatial frequency to determine focused pixel or region. However, in dynamic scenes we can't obtain focus information directly whether pixel or region is blurred or not. The reason is that the pixel or region in the same location of different source images may be contained of different contents due to camera movement or object motion. As well as traditional spatial domain methods cannot provide very accurate fusion results when pattern in source images become complex.

\section{TRADITIONAL MULI-FOCUS IMAGE FUSION TECHNIQUES}

Multi-focus image fusion Techniques broadly categorized as Transform Domain Fusion and Spatial Domain Fusion.

\subsection{Transform Domain Fusion}

In, transform domain fusion, the input images are decomposed based on transform coefficients. After that the fusion technique is applied and the fusion decision map is obtained. Finally, Inverse transformation applies on that decision map which produces fused image.

\subsubsection{DCT (Discrete Cosine Transform)}

In this method, Input images are divided in to nonOverlapping block having size NxN. DCT coefficients are computed for each block and fusion rules are applied to obtain fused DCT coefficients. Finally, IDCT applied on the fused coefficients to produce the final fused image [9].

\subsubsection{DWT (Discrete Wavelet Transform)}

In discrete wavelet transform (DWT) decomposition, the filters are specially designed so that successive layers of the 
pyramid only include details which are not already available at the preceding levels [9].

\subsubsection{Redundant discrete wavelet transform}

Redundant discrete wavelet transform (RDWT) is another variant of wavelet transform. It is used to overcome the shift variance problem of DWT. Input images are decomposed into three levels of RDWT decomposition using Daubechies filters on both the images in order to produce an approximate wavelet bands [10].

\subsubsection{Stationary wavelet transform}

Stationary Wavelet Transform (SWT) is similar to Discrete Wavelet Transform (DWT) but the only process of downsampling is suppressed that means the SWT is translationinvariant [11].

\subsubsection{Pyramid Method}

Pyramid method consists of set of lowpass or bandpass copy of an image. Each copy of an image represents the pattern information of a different scale. However, in pyramid method every level is a factor of two smaller as its predecessor, and higher level is focus on lower partial frequencies. This pyramid does not contain all information regarding to reconstruct the original image [15].

\subsection{Spatial Domain Fusion}

This type of fusion is directly applied to the input images which decrease the signal-to-noise ratio of the final fused image with the simple averaging.

\subsubsection{PCA (Principal Component Analysis)}

Principal component analysis is statistical technique which transform multivariate data with correlated variables into one with uncorrelated variables. These new variables are obtained as liner combination of the original variables[9].

\subsubsection{Averaging Method}

Averaging Method working as averaging every corresponding pixel of input images to obtain Resultant fused image.

$$
\mathrm{F}(x, y)=P(x, y)+Q(x, y) / 2
$$

Where, $\mathrm{F}(\mathrm{x}, \mathrm{y})$ is final fused image, $\mathrm{P}(\mathrm{x}, \mathrm{y})$ and $\mathrm{Q}(\mathrm{x}, \mathrm{y})$ are two input images [12].

\subsubsection{IHS (Intensity Hue Saturation) Fusion \\ Method}

IHS explains the popularity of perceptual colour space and overpowers the limitation of commonly used RGB colour space which does not relate intuitively to the attribute of human colour perception. Intensity means total amount of light that reaches the eye. Hue can be defined as the predominant wavelength of a color and saturation can be defined as purity of total amount of white light of a color [13].

\subsubsection{Brovey Method}

Brovey method is also known as color normalization transform hence it contains a red-green-blue (RGB) color transform method. The Brovey transformation was developed to avoid the drawbacks of the multiplicative method. It is the simplest method for combining data from different sensors. This method is used as combination of arithmetic operation and also uses spectral bands which are normalized before they are multiplied with the panchromatic image. It also retains the corresponding spectral feature of each pixel and transforms all the luminance information into a panchromatic image of high resolution [14].

\section{OTHER MULTI-FOCUS IMAGE FUSION TECHNIQUES 3.1 Novel Cross-Scale Fusion}

A Novel cross-scale fusion method is for multi-scale decomposition based on fusion of volumetric medical images considering both intra- and inter-scale consistencies. Fusion system passes information within each decomposition level so that the details of the source image is preserved expressing the artifacts. At initial step the information is passed from lower level to higher level, the membership of each fused coefficient is calculated using the passed information. The membership value is for guiding the details of the coefficient selection [3].

\subsection{Sparse Fusion of Image}

It is a new pan-sharpening method based on the compressive sensing theory. Pan-sharpening requires a low-resolution (LR) multispectral source image with $\mathrm{N}$ channels and another high resolution (HR) pan chromatic image. The aim is to increase spatial resolution of the source image while generating multispectral image. The Sparse FI algorithm reconstructs the HR multispectral image in an efficient way by ensuring both high spatial and spectral resolution with less spectral distortion. The method consists of three steps: dictionary learning, the HR image is low-pass filtered and down sampled that combined co-registration of different channels. Second Sparse Coefficient Estimation where each LR multispectral patch is combination of LR pan patches of the dictionary. Finally, the HR multispectral Image Reconstruction, where all the patches in all individual channels give the resultant pan image [4].

\subsection{Unsupervised Change detection}

The change detection algorithms play a significant role for various applications such as remote sensing, motion detection, environmental monitoring, medical diagnosis, damage assessment, agricultural surveys, surveillance and so on. An unsupervised change detection approach is proposed based on the concept of image fusion and kernel K-means clustering for retrieving better outcome of the synthetic aperture radar images. In this approach, the image can be generated by performing image fusion on mean-ratio and log-ratio techniques and discrete wavelet transform is applied for fusion. The non-linear clustering results in the degradation in false alarm rate and enhances the accuracy of the clustering process [5]

\subsection{Un-Decimated Wavelet Transform (UWT)}

Un-decimated wavelets transform (UWT) based fusion scheme, which splits the image decomposition process into two successive filtering operations using spectral factorization of the analysis filters. The UWT uses filter bank that decomposes one-dimensional signal to high pass coefficients. This approach overcomes the drawbacks of the conventional fusion process where the resultant image leads to the minimization of the unwanted spreading of coefficient values around overlapping image singularities complicates the feature selection process and lead to the reconstruction errors in the fused image. This approach independent of the fundamental fusion rule and reduces unwanted side effects such as ringing artifacts in the fused reconstruction [6].

\subsection{Wavelet based Fusion}

In, Application of tumor diagnosis of medical science, the exact size and position of tumor is a challenging issue to identify. To overcome this drawback, an efficient wavelet 
based algorithm is proposed for tumor detection which employed the complementary and redundant information from the image data. The approach efficiently utilizes the information retrieved from the input image in order to obtain a resultant fused image which increases the efficiency of tumor detection. In this approach, fusion is performed by considering the maximum value of the coefficients correspond to sharper brightness changes, results in the salient features visible. For multi-scale transform or multi resolution analysis, the images need to be decomposed into different levels like low-low, high-low, low-high, and high-high. This approach selects the appropriate decomposition levels by combining the salient features of source images overcome the problem of overlapping the neighboring features of the lower band signals [7].

\subsection{Kalman Fusion}

The image quality can be treated as a subjective impression of how well the image content is rendered or reproduced for enhancing the interpretation and image fusion is one of the major tools to improve the image quality. In the domain of remote sensing, where images can be acquired by multiple sources or by the same source in multiple acquisition contexts, appropriate fusion of the data require for obtaining better and consistent interpretation of the scene to retrieve overall perceptive of a phenomenon. To overcome these limitations, Kalman filter is applied for image fusion at pixel-level. The output image is formed with fusion of optimal estimates of pixel values for obtaining better Image quality to perceive [8].

\section{IMAGE MATTING IN MULTI- FOCUSED IMAGES}

Image matting is technique to accurately distinguish the foreground from background which has been widely used in

many applications, e.g. to obtain accurate focused object in video applications. In the model of image matting, the observed image $\mathrm{I}(\mathrm{x}, \mathrm{y})$ can be viewed as a combination of foreground $\mathrm{F}(\mathrm{x}, \mathrm{y})$ and background $\mathrm{B}(\mathrm{x}, \mathrm{y})$ :
$I(x, y)=\propto(x, y) F(x, y)+(1-\propto(x, y)) B(x, y)$

Where $\propto(\mathrm{x}, \mathrm{y})$ ranging from 0 to 1 is the foreground's opacities named as the alpha matte. $\propto(\mathrm{x}, \mathrm{y})=1$ or 0 means that $\mathrm{I}(\mathrm{x}, \mathrm{y})$ is in foreground or background, respectively. While $\propto(\mathrm{x}, \mathrm{y})$ is a fractional value between $(0,1)$, it means that these pixels are mixed by foreground and background [1].

The purpose of image matting is to find the exact alpha matt so that the foreground can be accurately illustrious from the background. Since there are three unknowns F, B, $\propto$ in Eq. (2), calculating the alpha matte a from a single image $\mathrm{I}$ is under constrained. Therefore, in most cases, the user is required to supply a trimap as the other input in addition to the original image.

\section{ENHANCE METHODOLOGY IN MULTI-FOCUS IMAGE FUSION \\ R-FCM (Rough-Fuzzy C-Means)}

R-FCM is a clustering Method in which Combination of fuzzy and rough sets are used. Combination of Fuzzy and Rough sets provides an significant route in reasoning with uncertainty. Both Fuzzy and Rough sets provide a mathematical structure to capture uncertainties which are connected with the data.

Cluster analysis is a technique for finding natural groups present in the data. It distinguished a given data set into a set of cluster in such a way that two object from the same cluster are as similar as possible and the objects from different cluster are as dissimilar as possible.

The Fuzzy sets membership function enables efficient handling of overlapping partitions, the concept of lower and upper approximation of rough sets deals with vagueness, uncertainty etc.

Image can be measured as an array of fuzzy singletons, after pre-filtering, each with a value of membership denoting the degree of brightness level according to membership function.

Table 1:Comparison of Multi-focus Image Fusion methods

\begin{tabular}{|c|c|c|}
\hline Methods & Advantages & Disadvantages \\
\hline Novel cross-scale fusion & $\begin{array}{l}\text { The fusion rules are effective and versatile } \\
\text { that produces high quality images }\end{array}$ & This is complex method to implement. \\
\hline Sparse Fusion of Images & $\begin{array}{l}\text { The Spares FI algorithm gives robust } \\
\text { performance against spectral model errors }\end{array}$ & $\begin{array}{l}\text { It does not assume an accurate spectral } \\
\text { model of the panchromatic image, and } \\
\text { hence, it is less sensitive to the model error } \\
\text { of the panchromatic image. }\end{array}$ \\
\hline Unsupervised change detection & $\begin{array}{l}\text { For detecting the changes this method is } \\
\text { more efficient compare to preexisting. }\end{array}$ & This method suffer from additive noise. \\
\hline Un-decimated wavelet transform (UWT) & $\begin{array}{l}\text { The approach achieves good } \\
\text { imperceptibility and robustness for fusion }\end{array}$ & $\begin{array}{l}\text { It is not exclusively found in multi-sensor } \\
\text { imagery }\end{array}$ \\
\hline Wavelet based Fusion & $\begin{array}{l}\text { The algorithm performs detection } \\
\text { efficiently for different decomposition } \\
\text { level }\end{array}$ & $\begin{array}{l}\text { It takes time for computation. It suffers } \\
\text { from color distortion problem. }\end{array}$ \\
\hline Kalman Fusion & $\begin{array}{l}\text { This provides better fused outcome for } \\
\text { multi-sensor satellite data images. }\end{array}$ & $\begin{array}{l}\text { Results are not so enormously exciting but } \\
\text { are convincing and satisfactory }\end{array}$ \\
\hline
\end{tabular}




\begin{tabular}{|c|c|c|}
\hline Principal Component Analysis (PCA) & $\begin{array}{l}\text { It is very simple to use in many } \\
\text { application. Images Fused by this method } \\
\text { have high spatial Quality. }\end{array}$ & $\begin{array}{l}\text { This method suffers from spectral } \\
\text { degradation. }\end{array}$ \\
\hline Averaging Method & $\begin{array}{l}\text { It is very simple method. Easy to } \\
\text { understand and implement. It works well } \\
\text { when input images are taken from same } \\
\text { sensor and contain additive Noise. }\end{array}$ & $\begin{array}{l}\text { It reduce the resultant image quality } \\
\text { consequently by introducing noise into } \\
\text { fused image. It leads to unwanted side } \\
\text { effects like reduce contrast. }\end{array}$ \\
\hline Intensity Hue Saturation (IHS) & $\begin{array}{l}\text { It provides good visual effect in fused } \\
\text { image. It is a simple method to merge the } \\
\text { images attributes. It gives best results in } \\
\text { remote sensing applications. }\end{array}$ & $\begin{array}{l}\text { It suffers from artifacts and noise which } \\
\text { tends to higher contrast. It produces } \\
\text { significant color distortion with respect to } \\
\text { the original image. }\end{array}$ \\
\hline Brovey Method & $\begin{array}{l}\text { It is simple and fast method used to merge } \\
\text { data from different sensors. It provide high } \\
\text { visual and high resolution multispectral } \\
\text { image. }\end{array}$ & $\begin{array}{l}\text { It suffers from spectral distortion. Brovey } \\
\text { method ignores the requirement of high } \\
\text { quality synthesis of spectral information. }\end{array}$ \\
\hline Discrete Cosine Transform (DCT) & $\begin{array}{l}\text { DCT provide efficient output. It reduces } \\
\text { the complexity and decomposed images } \\
\text { into series of waveform. }\end{array}$ & $\begin{array}{l}\text { This method leads to undesirable side } \\
\text { effect including blurring. }\end{array}$ \\
\hline Discrete Wavelet Transform (DWT) & $\begin{array}{l}\text { It provides good quality fused image. It } \\
\text { also provide better Signal to Noise Ratio. }\end{array}$ & $\begin{array}{l}\text { DWT produce image with shift variance } \\
\text { and additive noise. }\end{array}$ \\
\hline Redundant DWT (RDWT) & $\begin{array}{l}\text { RDWT is another variant of wavelet } \\
\text { transform and is used to overcome the } \\
\text { shift variance problem of DWT. }\end{array}$ & $\begin{array}{l}\text { It produces some additive noise at some } \\
\text { level of decomposition. }\end{array}$ \\
\hline Stationary Wavelet Transform (SWT) & $\begin{array}{l}\text { This method provides good result at level- } \\
2 \text { of decomposition. }\end{array}$ & $\begin{array}{l}\text { It is time consuming method compare to } \\
\text { DWT and implementation is difficult. }\end{array}$ \\
\hline Pyramid Method & $\begin{array}{l}\text { Pyramid Methods provide good visual } \\
\text { quality of an image for multi-focus } \\
\text { images. }\end{array}$ & $\begin{array}{l}\text { Contrast Pyramid could not retain } \\
\text { sufficient information from its source } \\
\text { images. Ratio Pyramid method suffers by } \\
\text { providing false information which never } \\
\text { existed in the original images. } \\
\text { Morphological Pyramid creates many false } \\
\text { edges. }\end{array}$ \\
\hline
\end{tabular}

\section{CONCLUSION}

In this paper, Various Traditional multi-focus image fusion Techniques and some novel multi-focus image fusion techniques have been studied. Paper also describes that how we can apply image matting in multi-focus image fusion. We can use R-FCM method to enhance the multi-focus image fusion process.

We can extend this work by implementing enhanced R-FCM method to fused Multi-Focus images in Dynamic Scene.

\section{REFERENCES}

[1] Shutao Li, Xudong Kang, Jianwen Hu, Bin Yang, "Image matting for fusion of multi-focus images in dynamic scenes", Journal of Information Fusion, pp. 147-162, (2013)

[2] Pramit Parekh, Nehal Patel, Robinson Macwan, Pritesh Kumar Prajapati, Sarita Visavalia, "Comparative Study and Analysis of Medical Image Fusion Techniques"(0975-8887)Volume.90-No.19,March(2014)
[3] Shen, Irene Cheng, and Anup Basu, "Cross-Scale Coefficient Selection for Volumetric Medical Image Fusion”, IEEE Transactions on Biomedical Engineering, Vol. 60, No. 4, pp. 1-10, (2013).

[4] Xiao Xiang Zhu and Richard Bamler, "A Sparse Image Fusion Algorithm With Application to Pan-Sharpening", IEEE Transaction on Geoscience and Remote Sensing, Vol. 51, No. 5, pp.2827-2836, (2013).

[5] K. Venkateswaran, N. Kasthuri and Arathy C. Haran V., "Unsupervised Change Detection using Image Fusion and Kernel K-Means Clustering", International Conference on Innovations In Intelligent Instrumentation, Optimization And Signal Processing, (2013).

[6] Andreas Ellmauthaler, CarlaL.Pagliari and Eduardo A. B. da Silva, "Multiscale Image Fusion Using the Undecimated Wavelet Transform With Spectral Factorization and Nonorthogonal Filter Banks", IEEE Transaction in Image Processing, Vol. 22, No.3, (2013). 
[7] Vivek Angoth, CYN Dwith and Amarjot Singh, "A Novel Wavelet Based Image Fusion for Brain Tumor Detection", International Journal of Computer Vision and Signal Processing, pp. 1-7, (2013).

[8] S. A. Quadri and Othman Sidek, "Pixel-Level Image Fusion using Kalman Algorithm", International Journal of Signal Processing, Image Processing and Pattern Recognition,Vol.6,(2013)

[9] Mr. Rajenda Pandit Desale, Prof. Sarita V. Verma, "Study and Analysis of PCA, DCT \& DWT based Image Fusion Techniques", International Conference on Signal Processing, Image Processing and Pattern Recognition [ICSIPR] (2013).

[10] S.Rajkumar, S.Kavitha, "Redundancy Discrete Wavelet Transform and Contourlet Transform for Multimodality Medical Image Fusion with Quantitative Analysis", 3rd International Conference on Emerging Trends in Engineering and Technology (2010).

[11] Mirajkar Pradnya P., Ruikar Sachin D., "Wavelet based Image Fusion Techniques", International Conference on Intelligent Systems and Signal Processing (ISSP) (2013).
[12] Prof. Keyur N. Brahmbhatt, Dr. Ramji M. Makwana, "Comparative study on image fusion methods in spatial domain", International journal of advanced research in engineering and technology (IJARET) (2013).

[13] KavitaTewari, Leena Shah, "Pixel Level Image Fusion Based on Spatial and Transform Domain", International Conference on Computer Science, Information and Technology, Pune, ISBN-978-93-81693-83-4 (2012)

[14] Rohan Ashok Mandhare, PragatiUpadhyay, Sudha Gupta, "Pixel-level image fusion using brovey transform and wavelet transform", International Journal of Advanced Research in Electrical, Electronics and Instrumentation Engineering(IJAREEIE), Vol. 2, Issue 6, June.(2013).

[15] FiroozSadjadi, "Comparative Image Fusion Analysais", Proceedings of the IEEE Computer Society Conference on Computer Vision and Pattern Recognition (CVPR'05) (2005). 